\title{
Derivations Commuting with Abelian Gauge Actions on Lattice Systems
}

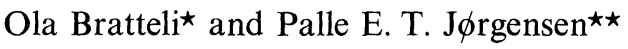 \\ 1 Mathematics Institute, University of Warwick, Coventry CV4 7AL, England \\ 2 Mathematics Institute, Aarhus University, Ny Munkegade, DK-8000 Aarhus C, Denmark
}

\begin{abstract}
Let $\tau$ be an action of a compact abelian group $G$ on a $C^{*}$-algebra $\mathscr{A}$, and assume that the fixed-point subalgebra $\mathscr{A}^{\tau}$ is an AF-algebra. We show that if $\delta$ is a closed ${ }^{*}$-derivation on $\mathscr{A}$ commuting with $\tau$, and the restriction of $\delta$ to $\mathscr{A}^{\tau}$ generates a one-parameter group of $*$-automorphisms, then $\delta$ itself is a generator. In particular, the result applies if $\tau$ is an infinite product action of $G$ on a UHF algebra. Furthermore, if in this situation $\delta_{1}$ and $\delta_{2}$ are two derivations both satisfying the hypotheses on $\delta$, and $\delta_{1}$ and $\delta_{2}$ have the same restriction to $\mathscr{A}^{\tau}$, then there exists a one-parameter subgroup of the action $\tau$ with generator $\delta_{0}$ such that $D\left(\delta_{1}\right) \cap D\left(\delta_{2}\right) \cap D\left(\delta_{0}\right)$ is a joint core for the three derivations, and $\delta_{2}$ $=\delta_{1}+\delta_{0}$ on this core.
\end{abstract}

\section{Introduction}

Let $\delta$ be a closed ${ }^{*}$-derivation with dense domain $D(\delta)$ in a $C^{*}$-algebra $\mathscr{A}$. Assume that $\delta$ commutes with a strongly continuous action $\tau$ of a compact abelian group $G$ as *-automorphisms on $\mathscr{A}$. It was shown in [4] that if $\delta$ vanishes identically on the fixed-point algebra $\mathscr{A}^{\tau}=\{A \in \mathscr{A}: \tau(g)(A)=A, g \in G\}$, then $\delta$ is the infinitesimal generator of a strongly continuous one-parameter group of *-automorphisms of $\mathscr{A}$. Briefly, we say that $\delta$ is a generator. By a simple perturbation argument, it follows that the assumption $\delta \mid \mathscr{A}^{\tau}=0$ may be weakened to the condition that $\delta \mid \mathscr{A}^{\tau}$ is inner. An example in [4] showed that it is not enough to assume that $\delta \mid \mathscr{A}^{\tau}$ is a generator on $\mathscr{A}^{\tau}$. In this example, $\mathscr{A}$ is abelian, and there is a geometric obstruction preventing $\delta$ from being a generator: Along the integral curves of the propagator, points burst into fibres, and conversely, fibres merge into points, in a finite time, [1].

On the other hand, Kishimoto and Robinson [15] showed that if one adds the assumption that $\mathscr{A}$ has an identity, and $\mathscr{A}^{\tau}(\gamma)^{*} \mathscr{A}^{\tau}(\gamma)=\mathscr{A}^{\tau}$ for all $\gamma \in \hat{G}$, where $\mathscr{A}^{\tau}(\gamma)=$

*Permanent address: Institute of Mathematics, N-7034 Trondheim-NTH, Norway

**Address from July 15, 1982: Department of Mathematics, University of Pennsylvania, Philadelphia, PA 19104, USA 
$\{X \in \mathscr{A}: \tau(g)(X)=\langle\gamma, g\rangle X$ for all $g \in G\}$, then $\delta$ is a generator if $\delta \mid \mathscr{A}^{\tau}$ is so. This spectral condition is, however, not satisfied in interesting examples like the standard gauge action of $\mathbb{T}^{1}$ on the CAR-algebra, [5]. This paper was motivated by this example, but we prove the slightly more general result that Kishimoto-Robinson's spectral condition can be replaced by the condition that each of the ideals $\mathscr{A}^{\tau}(\gamma)^{*} \mathscr{A}^{\tau}(\gamma)$ in $\mathscr{A}^{\tau}$ has an approximate identity consisting of projections. If, in particular, $\mathscr{A}^{\tau}$ is an AF algebra, then this condition is satisfied for all the ideals, [5]. It is interesting to note that if $\mathscr{A}^{\tau}$ is abelian and AF, then the geometric obstruction referred to above is ruled out for trivial reasons: all the closed ${ }^{*}$-derivations of $\mathscr{A}^{\tau}$ are zero, [3]. Also note that a small modification of [4, Example 6.1], where the string in the pinched torus is contracted to a point, shows that Kishimoto-Robinson's condition cannot be replaced by the condition that each of the ideals $\mathscr{A}^{\tau}(\gamma)^{*} \mathscr{A}^{\tau}(\gamma)$ in $\mathscr{A}^{\tau}$ is essential in the sense that it intersects any other non-zero ideal. Thus, when $\mathscr{A}$ is abelian, it is the total disconnectedness of the spectrum of the fixed point algebra which prevents geometric obstructions and make our proof work.

Our results are applicable to a slightly larger class of $C^{*}$-dynamical systems than the CAR algebra with the standard gauge action. Let $L$ be an index set, and for each $\imath \in L$, let $M_{\imath}$ be the full $n \times n$ matrix algebra. Let $\mathscr{A}=\otimes M_{\imath}$, and let $H$ be a compact abelian group acting on $M_{l}$ by an action $\tau^{l}$ which is independent of $l$. One can then associate a gauge action of first or second kind on $\mathscr{A}$. For the first kind, $G=H$ and $\tau(g)=\otimes_{\imath \in L} \tau^{\imath}(g)$, for the second kind, $G$ is the unrestricted direct product $\underset{\imath \in L}{\times} H$, and $\tau\left(\left(g_{l}\right)_{l}\right)=\bigotimes_{l \in L} \tau^{l}\left(g_{l}\right)$. In both cases, the canonical projection $P=\int_{G} d g \tau(g)$ onto the fixed point algebra $\mathscr{A}^{\tau}$ maps the finite sub-tensor products of $\mathscr{A}$ into themselves, and hence $\mathscr{A}^{\tau}$ is an AF-algebra.

For a gauge action of the second kind, Kishimoto-Robinson's (K-R) spectral condition is not satisfied unless it is satisfied on each factor $M_{\imath}$ for the group $H$, and this never happens if $\tau^{l}$ is implemented by a unitary action, i.e. the condition cannot be fulfilled if $H=\mathbb{T}^{d}$. For a gauge action of the first kind the situation is more complicated. If the index set $L$ is infinite, the fixed point algebra $\mathscr{A}^{\tau}$ is always prime, and if in addition $G$ is finite, then $\mathscr{A}^{\tau}$ is simple, i.e. the $\mathrm{K}-\mathrm{R}$ spectral condition is satisfied. If however $G$ is connected, then $\mathscr{A}^{\tau}$ is never simple, and the $\mathrm{K}$-R-condition is not valid [6], [19], see [21], [13] for further results in this direction.

This paper is organized as follows. In Sect. 2 we prove some results on smoothness of 1-cocycles. In Sect. 3 these results are combined with KishimotoRobinson's techniques from [15] to prove the main theorem. In Sect. 4, gauge actions of the first kind are analyzed in more detail, and Powers-Price's techniques from [17] are used to prove the result announced in the abstract.

\section{Automatic Smoothness of 1-cocycles}

Let $\delta$ be the generator of a strongly continuous one-parameter group $e^{t \delta}$ of $*_{\text {- }}$ automorphisms on a unital $C^{*}$-algebra $\mathscr{A}$. If $P$ is a skew-adjoint element in $\mathscr{A}$, define

$$
\delta^{P}(X)=\delta(X)+P X-X P=\delta(X)+\delta_{P}(X)
$$

for all $X \in D(\delta)$. Then $\delta^{P}$ is the generator of a one-parameter group $e^{t \delta^{P}}$ of 
*-automorphisms. There exists a map $t \rightarrow \Gamma_{t}^{P}$ from $\mathbb{R}$ into the unitary group of $\mathscr{A}$ such that

$$
e^{t \delta^{P}}(X)=\Gamma_{t}^{P} e^{t \delta}(X) \Gamma_{t}^{P *}
$$

for all $X \in \mathscr{A}, t \in \mathbb{R}$. The map can be taken to satisfy the 1-cocycle property $\Gamma_{t+s}^{P}=\Gamma_{t}^{P} e^{t \delta}\left(\Gamma_{s}^{P}\right)$. Here $\Gamma^{P}$ can be taken to be the unique solution of the differential equation

$$
\frac{d}{d t} \Gamma_{t}^{P}=\Gamma_{t}^{P} e^{t \delta}(P)
$$

with initial condition $\Gamma_{0}^{P}=\mathbb{\natural}$. Then $\Gamma^{P}$ also satisfy the equation

$$
\frac{d}{d t} \Gamma_{t}^{P}=e^{t \delta^{P}}(P) \Gamma_{t}^{P}
$$

and $\Gamma^{P}$ is given by the perturbation expansions

$$
\begin{aligned}
\Gamma_{t}^{P} & =\llbracket+\sum_{n \geqq 1} \int_{0}^{t} d t_{1} \int_{0}^{t_{1}} d t_{2} \ldots \int_{0}^{t_{n}-1} d t_{n} e^{t_{n} \delta}(P) \ldots e^{t_{1} \delta}(P) \\
& =\mathbb{}+\sum_{n \geqq 1} \int_{0}^{t} d t_{1} \int_{0}^{t_{1}} d t_{2} \ldots \int_{0}^{t_{n}-1} d t_{n} e^{t_{1} \delta^{P}}(P) \ldots e^{t_{n} \delta^{P}}(P),
\end{aligned}
$$

when $t \geqq 0$, see [8, Proposition 5.4.1.].

Each of the terms of these expansions involves a smoothing operation on $P$. This smoothing suffices to ensure that $\Gamma_{t}^{P}$ is always contained in the domain $D(\delta)$ of the generator $\delta$, even when $P$ is not contained in this domain. More precisely

Lemma 2.1. Adopt the assumptions and notation before this lemma. It follows that $\Gamma_{t}^{P} \in D(\delta)=D\left(\delta^{P}\right)$ and

$$
\begin{aligned}
\delta\left(\Gamma_{t}^{P}\right) & =\left(e^{t \delta^{P}}(P)-P\right) \Gamma_{t}^{P}, \\
\delta^{P}\left(\Gamma_{t}^{P}\right) & =\Gamma_{t}^{P}\left(e^{t \delta}(P)-P\right) .
\end{aligned}
$$

Hence one has

$$
\begin{aligned}
\frac{d \Gamma_{t}^{P}}{d t} & =\delta\left(\Gamma_{t}^{P}\right)+P \Gamma_{t}^{P} \\
& =\delta^{P}\left(\Gamma_{t}^{P}\right)+\Gamma_{t}^{P} P
\end{aligned}
$$

Proof. By [15, Theorem A1], $\mathscr{A}$ may be represented on a Hilbert space $\mathscr{H}$ such that $e^{t \delta}$ is covariant, i.e. there exists a strongly continuous unitary group $t \mapsto e^{t H}$ such that $e^{t \delta}(X)=e^{t H} X e^{-t H}$ for all $X \in \mathscr{A}$. Here $H$ is the skew-adjoint generator of the unitary group. The cocycle $\Gamma_{t}^{P}$ is then given by $\Gamma_{t}^{P}=e^{t(H+P)} e^{-t H},[8$, Corollary 5.4.2].

Assume first that $H$ is bounded, and then $\delta=\operatorname{ad}(H)$ extends to all of $\mathscr{L}(\mathscr{H})$. One has

$$
\begin{aligned}
\delta\left(\Gamma_{t}^{P}\right) & =\delta\left(e^{t(H+P)}\right) e^{-t H} \\
& =\int_{0}^{t} d s e^{s(H+P)} \delta(P) e^{(t-s)(H+P)} e^{-t H},
\end{aligned}
$$


see [18], or [7, Lemma 3.2.31]. Thus

$$
\begin{aligned}
\delta\left(\Gamma_{t}^{P}\right) & =\int_{0}^{t} d s e^{s(H+P)} \delta(P) e^{-s(H+P)} e^{t(H+P)} e^{-t H} \\
& =\int_{0}^{t} d s e^{s \delta^{P}}(\delta(P)) \Gamma_{t}^{P} \\
& =\int_{0}^{t} d s e^{s \delta^{P}}\left(\delta^{P}(P)\right) \Gamma_{t}^{P} .
\end{aligned}
$$

But $e^{s \delta^{P}}\left(\delta^{P}(P)\right)=(d / d s) e^{s \delta^{P}}(P)$, and hence $\delta\left(\Gamma_{t}^{P}\right)=\left(e^{t \delta^{P}}(P)-P\right) \Gamma_{t}^{P}$.

Assume next that $H=-H^{*}$ is unbounded. Using spectral theory, we may approximate $H$ by a sequence $\left(H_{n}\right)$ of bounded functions of $H$, i.e. each $H_{n}$ is bounded and skew-adjoint, and $H_{n} \psi \rightarrow H \psi$ for all $\psi \in D(H)$. It follows from the Trotter-Kato theorem in Kurtz' form [7, Theorem 3.1.28], that $e^{t H_{n}}$ and $e^{t\left(H_{n}+P\right)}$ converges strongly to $e^{t H}$ and $e^{t(H+P)}$. Hence

$$
\left(e^{t\left(H_{n}+P\right)} P e^{-t\left(H_{n}+P\right)}-P\right) e^{t\left(H_{n}+P\right)} e^{-t H_{n}}
$$

converges strongly to

$$
\left(e^{t \delta P}(P)-P\right) \Gamma_{t}^{P}
$$

It follows from the formula for $\delta\left(\Gamma_{t}^{P}\right)$ above that

$$
\left[H_{n}, e^{t\left(H_{n}+P\right)} e^{-t H_{n}}\right]
$$

converges strongly to $\left(e^{t \delta^{P}}(P)-P\right) \Gamma_{t}^{P}$, but at the same time it is clear from direct inspection that this expression converges as a bilinear form on $D(H) \times D(H)$ to $\left[H, \Gamma_{t}^{P}\right]$. It follows from [7, Proposition 3.2.55] that $\Gamma_{t}^{P} \in D(\delta)$ and

$$
\delta\left(\Gamma_{t}^{P}\right)=\left(e^{t \delta^{P}}(P)-P\right) \Gamma_{t}^{P}
$$

The remaining assertions of the lemma follows from the formuli

$$
\frac{d}{d t} \Gamma_{t}^{P}=e^{t \delta}(P) \Gamma_{t}^{P}=\Gamma_{t}^{P} e^{t \delta}(P)
$$

and $\delta^{P}=\delta+\delta_{P}$

\section{Derivations Commuting with Compact Abelian Actions}

Theorem 3.1. Let $\mathscr{A}$ be a $C^{*}$-algebra, $G$ a compact abelian group, and $\tau$ a continuous action of $G$ as ${ }^{*}$-automorphisms of $\mathscr{A}$. Assume that the fixed point algebra $\mathscr{A}^{\tau}$ for this action is an AF-algebra.

Let $\delta$ be a closed $*$-derivation on $\mathscr{A}$ satisfying

1. $\delta \tau(g)=\tau(g) \delta$ for all $g \in G$.

2. The restriction $\delta_{0}$ of $\delta$ to $\mathscr{A}^{\tau}$ is a generator. It follows that $\delta$ is a generator.

Remark. The assumption that $\mathscr{A}^{\tau}$ is an AF-algebra could be replaced by the 
hypothesis that each of the ideals $\overline{\mathscr{A}^{\tau}(\gamma)^{*} \mathscr{A}^{\tau}(\gamma)}$ in $\mathscr{A}^{\tau}$ has an approximate identity consisting of projections. This is the only property of AF-algebras used in the proof. In particular the proof applies if $\mathscr{A}^{\tau}$ is simple with unit, but this is already a consequence of $[15$, Theorem 1$]$.

Proof. If $\mathscr{A}$ does not have an identity, adjoin one and extend $\delta$ and $\tau$ in the obvious manner. The hypotheses in Theorem 3.1 are then still fulfilled and we may assume from now that $\mathscr{A}$ has an identity.

The strategy of the proof is as follows: We prove that the restriction of $\delta$ to each of the spectral subspaces $\mathscr{A}^{\tau}(\gamma)$ is a generator of a group of isometries. Actually, we restrict $\delta$ to subspaces of $\mathscr{A}^{\tau}(\gamma)$ of the form $\mathscr{A}^{\tau}(\gamma) P$, where $P$ are suitable smooth projections in an approximate identity for $\mathscr{A}^{\tau}(\gamma)^{*} \mathscr{A}^{\tau}(\gamma)$. We then perturb $\delta$ by an inner derivation $\delta_{-Q}=-\operatorname{ad}(Q)$ implemented by an element $Q \in \mathscr{A}^{\tau}$ to obtain a derivation $\delta^{-Q}=\delta+\delta_{-Q}$ such that $\delta^{-Q}(P)=0$, and hence $\delta^{-Q}$ leaves $\mathscr{A}^{\tau}(\gamma) P$ invariant. The derivation $\delta^{-Q}$ still satisfies the same hypotheses as $\delta$, in particular $\delta^{-Q} \mid \mathscr{A}^{\tau}$ is a generator. Kishimoto-Robinson introduced in [15] an explicit isometry between $\mathscr{A}^{\tau}(\gamma) P$ and a closed subspace of $\mathscr{A}^{\tau}$. (Actually this is a simplified account, and we have to work in a tensor product.) Transporting $\delta^{-Q}$ by this isometry, we get an operator on the closed subspace of $\mathscr{A}^{\tau}$ which is a bounded perturbation of $\delta^{-Q}$, and therefore is a generator there. It follows that $\delta^{-Q} \mid \mathscr{A}^{\tau}(\gamma) P$ is a generator. Perturbing back with a suitable 1 -cocycle in $\mathscr{A}$, we obtain the group generated by $\delta$.

Observation 1. If $\gamma \in \hat{G}$, there is a net $\left(P_{\alpha}\right)$ of projections in $\mathscr{A}^{\tau}(\gamma)^{*} \mathscr{A}^{\tau}(\gamma)$ such that:

1. Each $P \in\left(P_{\alpha}\right)$ is a finite sum $P=\sum_{k} X_{k}^{*} X_{k}$, where $X_{k} \in \mathscr{A}^{\tau}(\gamma) \cap D(\delta)$.

2. If $X \in \mathscr{A}^{\tau}(\gamma)$, then $\lim _{\alpha} X P_{\alpha}=X$, where the limit exists in norm.

Proof. $\overline{\mathscr{A}^{\tau}(\gamma)^{*} \mathscr{A}^{\tau}(\gamma)}$ is an ideal in $\mathscr{A}^{\tau}$, and as $\mathscr{A}^{\tau}$ is an AF-algebra, it follows that $\mathscr{A}^{\tau}(\gamma)^{*} \mathscr{A}^{\tau}(\gamma)$ has an approximate identity $\left(P_{\beta}^{\prime}\right)$ consisting of projections. There is a canonical projection from $\mathscr{A}$ onto $\mathscr{A}^{\tau}(\gamma)$ commuting with $\delta$, and hence $\mathscr{A}^{\tau}(\gamma) \cap D(\delta)$ is dense in $\mathscr{A}^{\tau}(\gamma)$. It follows, [11], [16], that for any $\varepsilon>0$ there are a finite number of elements $Y_{k} \in \mathscr{A}^{\tau}(\gamma) \cap D(\delta)$ such that

$$
\left\|P_{\beta}^{\prime}-\sum_{k} Y_{k}^{*} Y_{k}\right\|<\varepsilon
$$

If $Y=\sum_{k} Y_{k}^{*} Y_{k}$, then $Y \in \mathscr{A}^{\tau} \cap D(\delta)$, and as $P_{\beta}^{\prime}$ is a projection, the spectrum of $Y$ is contained in a small neighbourhood of the set $\{0,1\}$. Let $f \in C^{\infty}(\mathbb{R})$ be a function which is 0 in a suitable neighbourhood of 0 and $f(x)=x^{-1 / 2}$ in a suitable neighbourhood of 1 , such that $P_{\alpha}=P_{\beta, \varepsilon}=f(Y) Y f(Y)$ is a projection. Then $f(Y) \in D(\delta)$ by [20], or [7, Corollary 3.2.33] and hence $P_{\alpha} \in D(\delta)$. Furthermore, $\| P_{\beta}^{\prime}$ $-P_{\beta, \varepsilon} \|$ is dominated by a constant, only depending on $\varepsilon$, which vanishes as $\varepsilon \rightarrow 0$.

Thus, if the set of $(\beta, \varepsilon)$ is ordered by $(\beta, \varepsilon)<\left(\beta^{\prime}, \varepsilon^{\prime}\right)$ if $\beta<\beta^{\prime}$ and $\varepsilon^{\prime} \leqq \varepsilon$, the net $P_{\alpha}$ has the property that $\lim _{\alpha} X P_{\alpha}=X$, for all $X \in \overline{\mathscr{A}^{\tau}(\gamma)^{*} \mathscr{A}^{\tau}(\gamma)}$. But then $\lim _{\alpha} Y X P_{\alpha}=$ $Y X$, for $X \in \mathscr{A}^{\tau}(\gamma), Y \in \mathscr{A}^{\tau}(\gamma) \mathscr{A}^{\tau}(\gamma)^{*}$. But if $g$ is a non-negative real function such that 
$g(0)=0$ and $g(x)=1$ for $x \geqq \delta$, then $Y=g\left(X X^{*}\right) \in \mathscr{A}^{\tau}(\gamma) \mathscr{A}^{\tau}(\gamma)^{*}$ and

$$
\begin{aligned}
\left\|g\left(X X^{*}\right) X-X\right\| & \leqq\left\|\left(g\left(X X^{*}\right) X-X\right)\left(X^{*} g\left(X X^{*}\right)-X^{*}\right)\right\|^{1 / 2} \\
& =\left\|\left(g\left(X X^{*}\right)^{2}-2 g\left(X X^{*}\right)+\square\right) X X^{*}\right\|^{1 / 2} \\
& \leqq 2 \delta^{1 / 2}
\end{aligned}
$$

It follows from the relation above, by letting $\delta \rightarrow 0$, that $\lim X P_{\alpha}=X$, for all $X \in \mathscr{A}^{\tau}(\gamma)$.

If $X_{k}=Y_{k} f(Y)$, then $X_{k} \in \mathscr{A}^{\tau}(\gamma) \cap D(\delta)$ and $P_{\alpha}=\sum_{k} X_{k}^{*} X_{k}$. This ends the proof of Observation 1.

From now we fix a $\gamma \in \widehat{G}$ and a projection $P=\sum_{k=1}^{N} X_{k}^{*} X_{k}$, where each $X_{k} \in \mathscr{A}^{\tau}(\gamma) \cap D(\delta)$. Put $Q=\delta(P) P-P \delta(P), \quad \delta_{Q}(X)=Q X-X Q, \quad$ and $\delta^{-Q}(X)=$ $\delta(X)-\delta_{Q}(X)$ for $X \in D(\delta)$.

Then $Q$ is a skew-adjoint element in $\mathscr{A}^{\tau}$, and it follows that $\delta^{-Q}$ is a closed *-derivation satisfying the same hypotheses as $\delta$ in Theorem 3.1. Furthermore $\delta^{-Q}(P)=0,[14]$.

Let $\delta^{\prime}$ be the restriction of $\delta^{-Q}$ to $D\left(\delta^{\prime}\right)=\mathscr{A}^{\alpha}(\gamma) P \cap D(\delta)$. As $\delta^{-Q}(P)=0$ it follows that $\delta^{\prime}$ is a densely defined closed operator on $\mathscr{A}^{\alpha}(\gamma) P$.

Observation 2. The operator $\delta^{\prime}$ is the generator of a strongly continuous oneparameter group $S_{t}^{P}$ of bounded operators on $\mathscr{A}^{\alpha}(\gamma) P$.

Proof. We follow closely an argument from [15]. Recall that $P=\sum_{k=1}^{N} X_{k}^{*} X_{k}$. Consider the $C^{*}$-dynamical system $\left(\mathscr{A}_{N}=\mathscr{A} \otimes M_{N}, G, \bar{\tau}\right)$, where $M_{N}$ is the $N \times N$ matrix algebra, and $\bar{\tau}(g)=\tau(g) \otimes l$, where $l$ is the trivial action. Let $\bar{\delta}=\delta^{-Q} \otimes \imath$ with $D(\delta)=D(\delta) \otimes \imath$. Then $\bar{\tau}$ and $\bar{\delta}$ satisfy the same properties as $\tau$ and $\delta$. Define

$$
V=\left[\begin{array}{cccc}
X_{1} & 0 & \ldots & 0 \\
X_{2} & 0 & \ldots & 0 \\
\dot{\dot{X}_{N}} & 0 & \ldots & 0
\end{array}\right] \in \mathscr{A}_{N}^{\bar{\tau}}(\gamma) \cap D(\bar{\delta})
$$

Then

$$
V^{*} V=\left[\begin{array}{cccc}
P & 0 & \ldots & 0 \\
0 & 0 & \ldots & 0 \\
\vdots & & & \vdots \\
0 & 0 & \ldots & 0
\end{array}\right]
$$

and hence $V$ is a partial isometry, and $V V^{*}$ is a projection in $\mathscr{A}_{N}^{\bar{\tau}} \cap D(\bar{\delta})$. Furthermore

$$
\mathscr{A}_{N}^{\bar{\tau}}(\gamma) V^{*} V=\left[\begin{array}{llll}
\mathscr{A}^{\tau}(\gamma) P & 0 & \ldots & 0 \\
\mathscr{A}^{\tau}(\gamma) P & 0 & \ldots & 0 \\
\vdots & \vdots & & \vdots \\
\mathscr{A}^{\tau}(\gamma) P & 0 & \ldots & 0
\end{array}\right]
$$


Thus Observation 2 follows once we can show that the restriction $\bar{\delta}^{\prime}$, of $\bar{\delta}$ to $\mathscr{A}_{N}^{\bar{\tau}}(\gamma) V^{*} V$ is a generator.

To this end, we define an isometric isomorphism $\phi$ from the Banach space $\mathscr{A}_{N}^{\bar{\tau}}(\gamma) V^{*} V$ onto the Banach space $\mathscr{A}_{N}^{\bar{\tau}} V V^{*}$ by $\phi(A)=A V^{*}$, for $A \in \mathscr{A}_{N}^{\bar{\tau}}(\gamma) V^{*} V$. Then $\phi^{-1}(B)=B V$ for $B \in \mathscr{A}_{N}^{\bar{\tau}} V V^{*}$, and hence

$$
\phi \bar{\delta}^{\prime} \phi^{-1}(B)=\bar{\delta}^{\prime}(B V) V^{*}=\bar{\delta}(B V) V^{*}
$$

But as $B=B V V^{*}$, we have by the derivation property $\bar{\delta}(B)=\bar{\delta}(B V) V^{*}+B V \bar{\delta}\left(V^{*}\right)$. Thus $\phi \bar{\delta}^{\prime} \phi^{-1}(B)=\bar{\delta}(B)-B V \bar{\delta}\left(V^{*}\right)$, for all $B \in \mathscr{A}_{N}^{\bar{\tau}} V V^{*}$. Define an operator $\rho$ on $\mathscr{A}_{N}^{\bar{\tau}}$, by $\rho(B)=\bar{\delta}(B)-B V \bar{\delta}\left(V^{*}\right)$, for $B \in D(\bar{\delta}) \cap \mathscr{A}_{N}^{\bar{\tau}}$. Since $\bar{\delta}$ is the generator of a oneparameter group of automorphisms on $\mathscr{A}_{N}^{\bar{\tau}}$, and the operation of right multiplication by $-V \bar{\delta}\left(V^{*}\right)$ is a bounded operator on $\mathscr{A}_{N}^{\bar{\tau}}$, it follows that $\rho$ is the generator of a strongly continuous one-parameter group, [7, Theorem 3.1.33]. The restriction $\phi \bar{\delta}^{\prime} \phi^{-1}$ of $\rho$ to $\mathscr{A}_{N}^{\bar{\tau}} V V^{*}$ is then a generator, and as $\phi$ is an isometry, $\bar{\delta}^{\prime}$ is a generator. This completes the proof of Observation 2 .

Next, define a unitary cocycle $\Gamma_{t}^{Q}$ in $\mathscr{A}^{\tau}$ by

$$
\Gamma_{t}^{Q}=\rrbracket+\sum_{n \geqq 1} \int_{0}^{t} d t_{1} \int_{0}^{t_{1}} d t_{2} \cdots \int_{0}^{t_{n}-1} d t_{n} e^{t_{n} \delta-Q}(Q) \cdots e^{t_{1} \delta-Q}(Q),
$$

where $e^{t \delta-Q}$ here denotes the one-parameter group of *automorphisms generated by $\delta^{-Q}=\delta-\delta_{Q}$ on $\mathscr{A}^{\tau}$. Then $t \rightarrow \Gamma_{t}^{Q}$ is a continuous map into the unitaries in $\mathscr{A}^{\tau}$ satisfying the cocycle relation

$$
\Gamma_{t+s}^{Q}=\Gamma_{t}^{Q} e^{t \delta-Q}\left(\Gamma_{s}^{Q}\right)
$$

and the differential equation

$$
\frac{d \Gamma_{t}^{Q}}{d t}=\Gamma_{t}^{Q} e^{t \delta-Q}(Q)
$$

see Sect. 2.

Define a strongly continuous one-parameter family $T_{t}^{P}$ of maps from $\mathscr{A}^{\tau}(\gamma) P$ into $\mathscr{A}^{\tau}(\gamma)$ by

$$
T_{t}^{P}(X)=\Gamma_{t}^{Q} S_{t}^{P}(X) \Gamma_{t}^{Q *}
$$

Observation 3. If $X \in \mathscr{A}^{\tau}(\gamma) P \cap D(\delta)$, then $T_{t}^{P}(X) \in D(\delta)$ for all $t, t \rightarrow T_{t}^{P}(X)$ is differentiable and

$$
\frac{d T_{t}^{P}(X)}{d t}=\delta\left(T_{t}^{P}(X)\right)
$$

for all $t \in \mathbb{R}$.

Proof. As $X \in D(\delta)$ we have $X \in D\left(\delta^{-Q}\right)$ and hence $X \in D\left(\delta^{\prime}\right)$ and

$$
\frac{d}{d t} S_{t}^{P}(X)=\delta^{\prime}\left(S_{t}^{P}(X)\right)=\left(\delta-\delta_{Q}\right)\left(S_{t}^{P}(X)\right)
$$

It follows from Lemma 2.1 that $\Gamma_{t}^{Q} \in D\left(\delta^{-Q}\right)=D(\delta)$, and $(d / d t) \Gamma_{t}^{Q}=\delta^{-Q}\left(\Gamma_{t}^{Q}\right)+$ 
$Q \Gamma_{t}^{Q}=\delta\left(\Gamma_{t}^{Q}\right)+\Gamma_{t}^{Q} Q$. Thus $T_{t}^{P}(X) \in D(\delta), t \rightarrow T_{t}^{P}(X)$ is differentiable and

$$
\begin{aligned}
\frac{d T_{t}^{P}(X)}{d t}= & \frac{d \Gamma_{t}^{Q}}{d t} S_{t}^{P}(X) \Gamma_{t}^{Q^{*}}+\Gamma_{t}^{Q} \frac{d S_{t}^{P}(X)}{d t} \Gamma_{t}^{Q^{*}}+\Gamma_{t}^{Q} S_{t}^{P}(X)\left(\frac{d \Gamma_{t}^{Q}}{d t}\right)^{*} \\
= & \left(\delta\left(\Gamma_{t}^{Q}\right)+\Gamma_{t}^{Q} Q\right) S_{t}^{P}(X) \Gamma_{t}^{Q^{*}}+\Gamma_{t}^{Q}\left(\delta\left(S_{t}^{P}(X)\right)-Q S_{t}^{P}(X)\right. \\
& \left.+S_{t}^{P}(X) Q\right) \Gamma_{t}^{Q^{*}} \Gamma_{t}^{Q} S_{t}^{P}(X)\left(\delta\left(\Gamma_{t}^{Q^{*}}\right)-Q \Gamma_{t}^{Q^{*}}\right) \\
= & \delta\left(\Gamma_{t}^{Q}\right) S_{t}^{P}(X) \Gamma_{t}^{Q^{*}}+\Gamma_{t}^{Q} \delta\left(S_{t}^{P}(X)\right) \Gamma_{t}^{Q^{*}}+\Gamma_{t}^{Q} S_{t}^{P}(X) \delta\left(\Gamma_{t}^{Q^{*}}\right) \\
= & \delta\left(T_{t}^{P}(X)\right) .
\end{aligned}
$$

This proves Observation 3 .

Observation 4. If $X \in \mathscr{A}^{\tau}(\gamma) P$, then $\left\|T_{t}^{P}(X)\right\|=\|X\|$ for all $t \in \mathbb{R}$.

Proof. Let $e^{t \delta}$ again denote the automorphism group generated by the restriction of $\delta$ to $\mathscr{A}^{\tau}$. We first argue that

$$
T_{t}^{P}(X)^{*} T_{t}^{P}(X)=e^{t \delta}\left(X^{*} X\right)
$$

for all $t \in \mathbb{R}$. Assume first $X \in \mathscr{A}^{\tau}(\gamma) P \cap D(\delta)$. It follows from Observation 3 and the derivation property that the following calculation is valid

$$
\begin{aligned}
& T_{t}^{P}(X)^{*} T_{t}^{P}(X)-e^{t \delta}\left(X^{*} X\right) \\
& =\int_{0}^{t} d s \frac{d}{d s}\left\{e^{(t-s) \delta}\left(T_{s}^{P}(X)^{*} T_{s}^{P}(X)\right)\right\} \\
& =\int_{0}^{t} d s e^{(t-s) \delta}\left(-\delta\left(T_{s}^{P}(X)^{*} T_{s}^{P}(X)\right)+\delta\left(T_{s}^{P}(X)^{*}\right) T_{s}^{P}(X)\right. \\
& \left.\quad+T_{s}^{P}(X)^{*} \delta\left(T_{s}^{P}(X)\right)\right) \\
& =\int_{0}^{t} d s e^{(t-s) \delta}(0)=0
\end{aligned}
$$

Thus

$$
\left\|T_{t}^{P}(X)\right\|^{2}=\left\|T_{t}^{P}(X)^{*} T_{t}^{P}(X)\right\|=\left\|e^{t \delta}\left(X^{*} X\right)\right\|=\left\|X^{*} X\right\|=\|X\|^{2} .
$$

As $P \in D(\delta), \mathscr{A}^{\tau}(\gamma) P \cap D(\delta)$ is dense in $\mathscr{A}^{\tau}(\gamma) P$, and as each $T_{t}^{P}$ is bounded, Observation 4 follows.

Observation 5. The restriction $\delta_{\gamma}$ of $\delta$ to $\mathscr{A}^{\tau}(\gamma)$ is the generator of a strongly continuous one-parameter group of isometries on $\mathscr{A}^{\tau}(\gamma)$.

Proof. The operators $\pm \delta_{\nu}$ are dissipative by [4, Lemma 4.3], and by the LumerPhillips theorem it suffices to prove that $(\lambda-\delta)\left(D\left(\delta_{\gamma}\right)\right)$ is dense in $\mathscr{A}^{\tau}(\gamma)$ for a positive and a negative real number $\lambda$, see [7, Theorem 3.1.16]. This follows from the differential equation in Observation 3 as in [9, page 360-361], or as follows: Assume 
that $X \in \mathscr{A}^{\tau}(\gamma) P \cap D(\delta)$, where $P$ is as in Observation 1, and assume that $\lambda>0$. We will show that $X \in R\left(\lambda-\delta_{\gamma}\right)$. Define

$$
Y=\int_{0}^{\infty} d t e^{-\lambda t} T_{t}^{P}(X)
$$

where $T^{P}$ is defined before, Observation 3. The integral converges because of Observation 4, and Observation 3 implies that $Y \in D(\delta)$ with

$$
\begin{aligned}
\delta(Y) & =\int_{0}^{\infty} d t e^{-\lambda t} \delta\left(T_{t}^{P}(X)\right)=\int_{0}^{\infty} d t e^{-\lambda t} \frac{d}{d t}\left(T_{t}^{P}(X)\right) \\
& =\int_{0}^{\infty} d t \frac{d}{d t}\left(e^{-\lambda t} T_{t}^{P}(X)\right)+\lambda \int_{0}^{\infty} d t e^{-\lambda t} T_{t}^{P}(X) \\
& =-X+\lambda Y .
\end{aligned}
$$

Thus $X=(\lambda-\delta) Y$ and $X \in R(\lambda-\delta)$. It now follows from Observation 1 that $R(\lambda-\delta)$ is dense. This ends the proof of Observation 5 .

Theorem 3.1 is now an immediate consequence of Observation 5 and [15, Proposition 2].

\section{Gauge Actions of the First Kind}

In this section, which is largely independent of the previous one, we will consider the following problem: Let $\mathscr{A}$ be a $C^{*}$-algebra, $\tau$ an action of a compact abelian group on $\mathscr{A}, \delta$ a generator on the fixed point algebra $\mathscr{A}^{\tau}$. Assume that $\delta$ has closed densely defined extensions to $\mathscr{A}$ which commute with $\tau$, and let $\delta_{1}, \delta_{2}$ be two such extensions. What is the relation between $\delta_{1}$ and $\delta_{2}$ ?

We will actually consider this problem in a special setting.

Theorem 4.1. Let $\mathscr{A}$ be a UHF-algebra of Glimm type $n^{\infty}$, i.e. $\mathscr{A}=\otimes_{\imath \in L} M_{\imath}$, where the index set $L$ is infinite and each $M_{\imath}$ is a full $n \times n$-matrix algebra $M$. Let $G$ be a compact abelian group acting on $M$ and let $\tau$ be the corresponding infinite tensor product action of $G$ on $\mathscr{A}$. Let $\alpha$ be an automorphism of $\mathscr{A}$ such that

$$
\alpha \tau(g)=\tau(g) \alpha \quad \text { for all } g \in G,
$$

and

$$
\alpha(X)=X \quad \text { for all } X \in \mathscr{A}^{\tau} \text {. }
$$

It follows that there exists a $g \in G$ such that $\alpha=\tau(g)$. In particular, if $\delta_{1}, \delta_{2}$ are

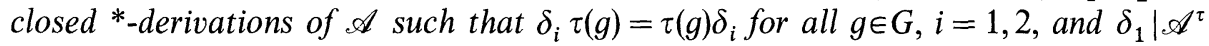
$=\delta_{2} \mid \mathscr{A}^{\tau}$, and $\delta_{1} \mid \mathscr{A}^{\tau}$ is a generator on $\mathscr{A}^{\tau}$, then there exists a one-parameter subgroup of the action $\tau$ with generator $\delta_{0}$, such that $D\left(\delta_{2}\right) \cap D\left(\delta_{1}\right) \cap D\left(\delta_{0}\right)$ is a joint core for the three derivations and $\delta_{2}=\delta_{1}+\delta_{0}$ on this core.

Remark. Actually we prove the slightly stronger result that the groups $e^{t \delta} i$ mutually commute for $i=0,1,2$, and the relation $e^{t \delta 2}=e^{t \delta 0} e^{t \delta 1}$ is valid for all $t \in \mathbb{R}$. 
Lemma 4.2. Adopt the same hypotheses on $\mathscr{A}, \tau$ as in Theorem 4.1, but $G$ may be any subgroup of $\operatorname{Aut}(\mathrm{M})$. Let $(\mathscr{H}, \pi, \Omega)$ be the cyclic representation of $\mathscr{A}$ defined by the unique trace-state $\omega$ on $\mathscr{A},\left[7\right.$, Definition 2.3.18]. It follows that $\pi\left(\mathscr{A}^{\tau}\right)^{\prime} \cap \pi(\mathscr{A})^{\prime \prime}=\mathbb{C l}$, and in particular $\pi\left(\mathscr{A}^{\tau}\right)^{\prime \prime}$ is a factor.

Remark. It is known that the restriction of $\omega$ to $\mathscr{A}^{\tau}$ is a factor state; for a sharp result in this direction, see [21, Theorem 4 in Sect. III]. As the closed cyclic subspace generated by $\mathscr{A}^{\tau}$ in $\mathscr{H}$ is a proper subspace of $\mathscr{H}$, Lemma 4.2 requires a separate proof.

Proof. Although this probably could be proved using techniques from [12], we will use an argument based on an idea from [17]. Let $S(\infty)$ be the group of all finite permutations of the index set $L$. There is a canonical action $\alpha$ of $S(\infty)$ on $\mathscr{A}=\otimes M_{1}$, and, as shown in [17], there is a unitary representation $s \in S(\infty) \rightarrow U(s) \in \mathscr{A}^{\tau}$ such that $\alpha(s)=\operatorname{Ad}(U(s))$ for all $s \in S(\infty)$.

As $\omega$ is invariant under all automorphisms of $\mathscr{A}, \omega$ is $\alpha$-invariant. As $\omega$ is the product of the normalized traces on $M_{\imath}$ for all $l$, it follows by approximating with finite tensors that $\omega$ is clustering with respect to the action $\alpha$, i.e. for any $A, B \in \mathscr{A}$ and $\varepsilon>0$, there exists an $s \in S(\infty)$ such that $|\omega(A \alpha(s)(B))-\omega(A) \omega(B)|<\varepsilon$. It follows that $\omega$ is external among the $\alpha$-invariant states [7, Theorems 4.3.22 and 4.3.20]. As $\omega$ is a trace, the cyclic vector $\Omega$ is separating for $\pi(\mathscr{A})^{\prime \prime}$. If $U_{\omega}$ is the unitary representation of $S(\infty)$ on $\mathscr{H}$, defined by

$$
U_{\omega}(s) \pi(A) \Omega=\pi(\alpha(s)(A)) \Omega
$$

for all $A \in \mathscr{A}, s \in S(\infty)$, then it follows from extremal invariance of $\omega$ that

$$
\pi(\mathscr{A})^{\prime \prime} \cap U_{\omega}(S(\infty))^{\prime}=\mathbb{C} \mathbb{l},
$$

$\left[7\right.$, Theorem 4.3.20]. As each $U_{\omega}(s)$ implements the same automorphism of $\pi(\mathscr{A})^{\prime \prime}$ as $\pi(U(s))$, and $\pi$ is a factor representation, it follows that there exists a unitary operator $j(\pi(U(s))) \in \pi(\mathscr{A})^{\prime}$ such that

$$
U_{\omega}(s)=\pi(U(s)) j(\pi(U(s)))
$$

and hence

$$
\pi(\mathscr{A})^{\prime \prime} \cap \pi(U(S(\infty)))^{\prime}=\pi(\mathscr{A})^{\prime \prime} \cap U_{\omega}(S(\infty))^{\prime}=\mathbb{C l} .
$$

But as $U(S(\infty)) \in \mathscr{A}^{\tau}$ it follows finally that

$$
\pi(\mathscr{A})^{\prime \prime} \cap \pi\left(\mathscr{A}^{\tau}\right)^{\prime}=\mathbb{C l} \text {. }
$$

Proof of Theorem 4 1. Let $\alpha$ be an automorphism of $\mathscr{A}$ commuting with $\tau$ such that $\alpha \mid \mathscr{A}^{\tau}=$ id. As the trace state $\omega$ on $\mathscr{A}$ is $\alpha$-and $\tau$-invariant, $\alpha$ and $\tau$ extends by $\sigma$-weak closure to $\pi(\mathscr{A})^{\prime \prime}$, and as the projection $P(X)=\int_{G} d g \tau(g)(X)$ extends by $\sigma$-weak closure to $\pi(\mathscr{A})^{\prime \prime}$, we have that $\pi\left(\mathscr{A}^{\tau}\right)^{\prime \prime}$ is the fixed point algebra for the extended $\tau$. Hence the extended $\alpha$ restricts to the identity on this fixed point algebra, and the extended $\alpha$ commutes with the extended $\tau$. But Lemma 4.2 implies that $\pi\left(\mathscr{A}^{\tau}\right)^{\prime \prime}$ is a 
factor, and it follows from Robert's version of Pontryagins duality theorem that there exists a $g \in G$ such that $\alpha=\tau(g)$. (A general version of Roberts's theorem is proved in [2, Appendix C], the special version used here can also be found in [10].)

Finally, let $\delta_{1}, \delta_{2}$ be two closed *-derivations on $\mathscr{A}$ commuting with $\tau$ such that $\delta_{1}\left|\mathscr{A}^{\tau}=\delta_{2}\right| \mathscr{A}^{\tau}$ is a generator on $\mathscr{A}^{\tau}$. It follows from Theorem 3.1 that $\delta_{1}, \delta_{2}$ generates groups $e^{t \delta_{1}}, e^{t \delta_{2}}$ on $\mathscr{A}$, and then $e^{t \delta_{1}}\left|\mathscr{A}^{\tau}=e^{t \delta_{2}}\right| \mathscr{A}^{\tau}$. Hence, by the first part of the theorem, there exists for each $t \in \mathbb{R}$ an element $g(t) \in G$ such that $e^{t \delta_{2}}=\tau(g(t)) e^{t \delta_{1}}$. By $[2$, Appendix $B]$ we may assume that $G$ acts faithfully, and hence $g(t)$ is unique. Here $\tau(g(s))$ commutes with $e^{t \delta_{1}}$ and $e^{t \delta_{2}}$ by assumption, for $s, t \in \mathbb{R}$ and hence $t \rightarrow g(t)$ is a one-parameter group. Also $t \rightarrow \tau(g(t))=e^{t \delta_{2}} e^{-t \delta_{1}}$ is strongly continuous and it has a generator $\delta_{0}$ which is a closed densely defined *-derivation. As $e^{s \delta_{0}}$ and $e^{t \delta_{1}}$ commute for all $s, t$, we get a strongly continuous representation by $\mathbb{R}^{2}$ by $(s, t)$ $\rightarrow e^{s \delta_{0}} e^{t \delta_{1}}$. The $C^{1}$-vectors $D\left(\delta_{0}\right) \cap D\left(\delta_{1}\right)$ for this representation are invariant under both $e^{s \delta_{0}}$ and $e^{t \delta_{1}}$. It follows from $e^{t \delta_{2}}=e^{t \delta_{0}} e^{t \delta_{1}}$ that these vectors are contained in $D\left(\delta_{2}\right)$ and are invariant under $e^{t \delta_{2}}$. Hence these vectors are a core for both $\delta_{0}, \delta_{1}$ and $\delta_{2},\left[7\right.$, Corollary 3.1.7]. Thus $D\left(\delta_{0}\right) \cap D\left(\delta_{1}\right) \cap D\left(\delta_{2}\right)$ is a joint core for $\delta_{0}, \delta_{1}$ and $\delta_{2}$.

It follows from $e^{t \delta_{2}}=e^{t \delta_{0}} e^{t \delta_{1}}$ that $\delta_{2}=\delta_{1}+\delta_{0}$ on this core.

Acknowledgements. We are indebted to David E. Evans for pointing out that Powers-Price's techniques from [17] are relevant for the proof of Lemma 4.2. The second author gratefully acknowledges fruitful discussions with F. Goodman, R. T. Powers and G. Price during the Iowa meeting in April 1982. The university of Aarhus provided the travel support which made this collaboration possible. The second author's wife generously provided the food and hospitality which made the work pleasant. The first author was supported by a Science and Engineering Research Council Senior Visiting Fellowship at University of Warwick.

\section{References}

1. Aizenman, M.: On vector fields as generators of flows: A counterexample to Nelsons conjecture. Ann. Math. 107, 287-296 (1978)

2. Araki, H., Haag, R., Kastler, D., Takesaki, M.: Extension of KMS states and chemical potential. Commun. Math. Phys. 53, 97-134 (1977)

3. Batty, C. J. K.: Unbounded derivations of commutative $C^{*}$-algebras. Commun. Math. Phys. 61, 419-425 (1978)

4. Bratteli, O., J $\phi$ rgensen, P. E. T.: Unbounded derivations tangential to compact groups of automorphisms. J. Funct. Anal. (to appear)

5. Bratteli, O.: Inductive limits of finite-dimensional $C^{*}$-algebras. Trans. Am. Math. Soc. 171, 195-234 (1972)

6. Bratteli, O.: Crossed products of UHF-algebras by product type actions. Duke Math. J. 46, 1-23 (1979)

7. Bratteli, O., Robinson, D. W.: Operator algebras and quantum statistical mechanics I. New York, Heidelberg, Berlin: Springer 1979

8. Bratteli, O., Robinson, D. W.: Operator algebras and quantum statistical mechanics II. New York, Heidelberg, Berlin: Springer 1981

9. Bratteli, O., Kishimoto, A.: Generation of semigroups and two-dimensional quantum lattice systems. J. Funct. Anal. 35, 344-368 (1980)

10. Bratteli, O., Evans, D. E.: Dynamical semigroups commuting with compact abelian actions. Warwick preprint (1982)

11. Brown, L. G.: Stable 1somorphism of hereditary subalgebras of $C^{*}$-algebras. Pac. J. Math. 71, 335348 (1977) 
12. Connes, A.: Une classification des facteurs de type III. Ann. Sci. Ecole Norm. Sup. 6, 133-252 (1973)

13. Handelman, D., Rossmann, W.: Product type actions of finite and compact groups. Preprint

14. Kaplansky, I.: Modules over operator algebras. Am. J. Math. 75, 839-859 (1953)

15. Kishimoto, A., Robinson, D. W.: On unbounded derivations commuting with a compact group of *automorphisms. New South Wales preprint (1981)

16. Kishimoto, A., Takai, H.: Some remarks on $C^{*}$-dynamical systems with a compact abelian group. Publ. R.I.M.S. Kyoto Univ. 14, 383-397 (1978)

17. Powers, R. T., Price, G.: Derivations vanishing on $S(\infty)$. Commun. Math. Phys. 84, 439-447 (1982)

18. Powers, R. T.: A remark on the domain of an unbounded derivation of a $C^{*}$-algebra. J. Funct. Anal. 18, 85-95 (1975)

19. Rosenberg, J.: Appendix to O. Bratteli's paper on: Crossed products of UHF algebras. Duke Math. J. 46, 25-26 (1979)

20. Sakai, S.: On one parameter groups of *-automorphisms on operator algebras and the corresponding unbounded derivations. Am. J. Math. 98, 427-440 (1976)

21. Wassermann, A. J.: Automorphic actions of compact groups on operator algebras. University of Pennsylvania Thesis (1981)

Communicated by H. Araki

Received June 18, 1982 